\title{
The Beginnings of Optoelectronic Integrated Circuits-A Personal Perspective
}

\author{
Amnon Yariv, Life Fellow, IEEE
}

Invited Paper

\begin{abstract}
The early work (1960s, 1970s) on Integrated Optics at the Bell Telephone Laboratories and at the California Institute of Technology (Caltech), with which the author was involved, is described from a personal perspective.
\end{abstract}

Index Terms-Integrated optics.

W HEN asked by my colleague Alan Willner to contribute an article for this issue on Integrated Optics I hesitated before accepting - mostly due to the magnitude of the task but also because I have not followed closely the more recent developments. I accepted, however, with the provision that I will limit my reminiscences to the early days of the field and specifically to the part of the field with which I was involved first at the Bell Telephone Laboratories and then at Caltech and which deals with what is now known as Optoelectronic Integrated Circuits (OEICs) which involves monolithic integration of optical devices (lasers, waveguides) and electronics (transistors, modulators) devices.

The readers who wish to delve more deeply into those early days could consult: 1) P. K. Tien, "Integrated optics and new wave phenomena in optical waveguides," Rev. of Mod. Phys., Vol. 49, No. 2, Apr. 1977 or 2) M. Hirano, "OEICs," Optoelectronics-Devices and Technologies, Vol. 2, No. 2, Dec. 1987.

Since issues of priority and historical occurrence invariably creep into any article that reviews a relatively long period and describes the efforts of many individuals I decided to adhere to a simple rule: unless there is strong evidence to the contrary, it is assumed that the earlier publication implies historical precedence. It is understood, of course, that the temporal resolution of this approach is no better than a few months.

I landed in Newark, NJ in January 1959 with a newly minted $\mathrm{Ph} . \mathrm{D}$. degree in electrical engineering from UC Berkeley and joined the Electronics Research Laboratory at the Bell Telephone Laboratories (the "Labs") in Murray Hill. The laboratory was headed at that time by John Pierce of the Traveling wave tube and, later, communication satellites fame.

One of the most exciting aspects of the work at Bell was the association with talented colleagues, most of them new recruits like myself. Our small department included J. P. Gordon who

Manuscript received January 18, 2008.

The author is with the Applied Physics and Electrical Engineering Department, California Institute of Technology, Pasadena, CA 91125 USA (e-mail: ayariv@caltech.edu).

Digital Object Identifier 10.1109/JLT.2008.923630

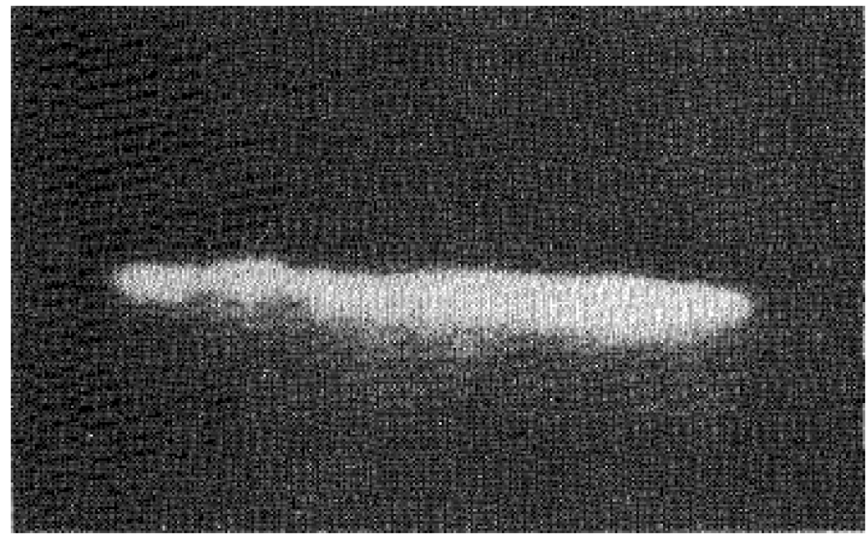

Fig. 1. Photograph of the front surface of the diode in which the bright region corresponds to light confined by the dielectric-waveguide effect.

as Townes' student at Columbia made the first Ammonia laser and years later would make with Herman Haus major contributions to the soliton area, Kumar Patel discovering new gas lasers by the week, and Gary Boyd and Herwig Kogelnik with their now classic work on Gaussian optical beam modes. I would also interact closely with J. Giordmaine (inventor of crystal phase matching), Ivan Kaminow (microwave modulation of lasers), P. K. Tien, and J. Geusic with his newly invented Nd:YAG laser.

I spent a couple of years or so working on a number of projects including a search for new laser materials and working on some isoteric noise problems. One interesting outcome of a collaboration with W. H. Louisell of the Labs and with Tony Siegman (at Stanford) was a quantum mechanical formalism for nonlinear optics [1]. This formalism has become the starting point for quantum analyses of a variety of 3 photon and 4 photon parametric processes including optical squeezing. This relatively relaxed period came to an end sometime in 1963.

A group of researchers at the G. E. Laboratories (Hall, Fenner, Kingsley, Soltys, and Carlson) and an IBM group (Nathan, Dumke, Burns, Dills, and Lasher) announced in 1962, simultaneously, the operation of a semiconductor laser [2], [3]. A task force consisting of Rogerio Leite, Barry Cohen, Walter Bond, and myself decided to repeat the G. E. and IBM work and evaluate the potential of the new invention which somehow caught the Bell Laboratories by surprise. In a matter of a few weeks we had GaAs lasers oscillating at liquid $\mathrm{N}_{2}$ temperatures. The main experimental evidence for laser oscillation was a distinct, now familiar, threshold behavior in the light power versus injection current characteristics. Also a simultaneous 
appearance of a bright stripe $1 \mu \mathrm{m} \times 6 \mu \mathrm{m}$ on the output cleaved facet of the laser is shown in Fig. 1.

In trying to explain the origin of this stripe we came up with the model of a fortuitous dielectric waveguide straddling the laser diode p-n junction [4], [5]. This waveguide was responsible for confining the emitted laser radiation where it was amplified by induced electron-hole recombination. Without this confinement the spread of the laser beam would have resulted in a laser threshold orders of magnitude bigger than the one observed. A similar conclusion about the existence of dielectric waveguiding near (passive, nonlasing) p-n junctions was reached by Ashkin and Gershenzon, also at the Labs, based on their experiments in nonlasing GaP p-n junctions [6]. (An aside: Although we recognized the all-important role of dielectric waveguiding in drastically bringing down the threshold current, and thus making lasing possible, of the SC lasers, it did not occur to us at the time to deliberately engineer a strong and narrow waveguide in the active junction region that would make continuous-wave room temperature laser oscillation possible. That major development would be done years later, by Kroemer and Alferov using hetero-epitaxy. This work would earn them the Nobel Prize in physics in 2000 [7].)

In 1964, I left the Bell Laboratories and joined the Electrical Engineering Department at Caltech. For my first project I decided to continue investigating the phenomenon of optical guiding in semiconductors. Instead of relying on the accidental and not fully understood waveguiding we encountered at the GaAs laser p-n junction we decided to try and fabricate the waveguide deliberately. The task fell to one of my first group of students, David Hall. David's project was to look for optical guiding in a surface of a GaAs slab. The waveguiding will be due to a lowering of the dielectric constant in the low-resistivity substrate relative to that of the carrier-depleted surface layer by

$$
\delta \varepsilon=\frac{N e^{2}}{m^{* \varepsilon}}
$$

where $N$ is the carrier density in the high-conductivity substrate and $m^{*}$ is the effective mass of the carriers. It so happened that such structures were being fabricated at the time by researchers working on the Gunn effect, and we hoped that some such device would fit our requirements.

David Hall spent the better part of two years looking for guiding in many crystal samples which were donated by various laboratories, with no success. Sometime toward the spring of 1969, we agreed that, if by the end of the summer we could not observe guiding, then David would abandon the experiment and move on to some "safe" project. Early that summer, David went home to Hartford, CT, on vacation and there discovered a new, yet untapped by us, source of crystals at the United Aircraft Research Laboratories. If I am not mistaken, these GaAs crystals were grown by Dr. Alexander Shuskus.

By the end of the summer Dave would process the crystals into appropriate samples and almost immediately, working with Elsa Garmire, who in the meantime joined us as a postdoctoral fellow, observed waveguiding. My own excitement was exceeded only by that of Dave to whom the bright strip of light on

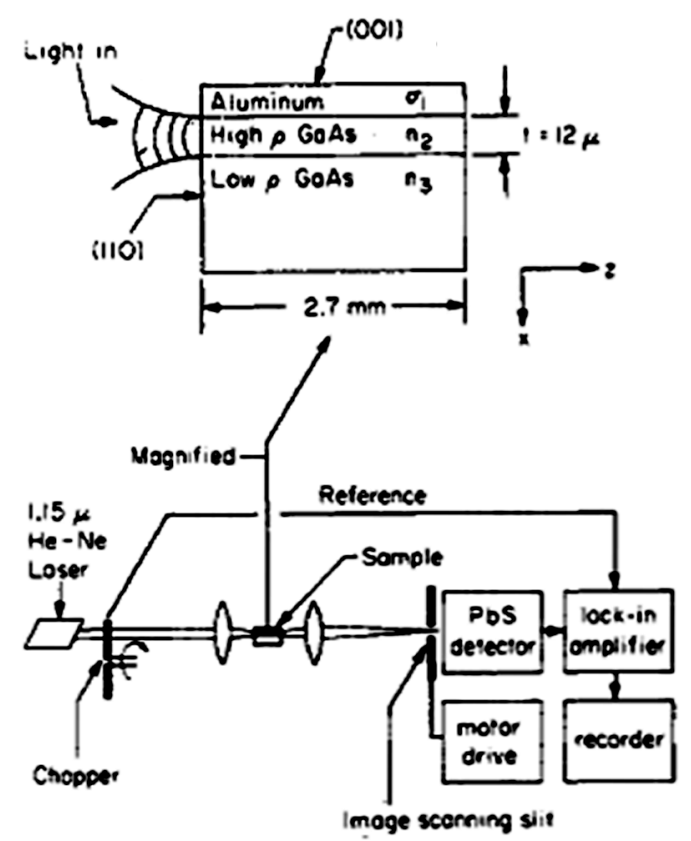

Fig. 2. (Upper) Epitaxial GaAs waveguide electrooptic modulator. (Lower) The experimental setup for observing the modulation.

the image converter signified not only a successful experiment, but also a good part of his Ph.D. thesis.

Fig. 2 is a reproduction from the 1970 paper [8] describing the work. You will note that in addition to the waveguide, we also deposited a metal electrode on the crystal. This small but significant addition to the experiment made it possible to control the index of refraction in the guiding region by applying a (reverse) voltage between the substrate and the metal. The resulting depletion layer (Schottky barrier) electric field acting through the electrooptic effect of GaAs changes the dielectric constant of the guiding layer by

$$
\delta \varepsilon=\varepsilon_{0} n^{4} r_{41} E
$$

where $r_{41}$ is the electrooptic coefficient of GaAs, $n$ its refraction, and $E$ the electric field.

The total change of the dielectric constant of the guiding channel $\delta \varepsilon$ is thus the sum of (1) and (2) so that with a proper choice of the sign of $E, \delta \varepsilon$ can be made to drop below the threshold value (for guiding) and the guiding would (and did) disappear. We thus had a voltage-controlled dielectric waveguide which could be used as a light modulator. We have thus demonstrated the first guided-wave electrooptic modulation. The use of electrooptic waveguide modulators in $\mathrm{LiNbO}_{3}$, an invention of my colleague Ivan Kaminow at the Holmdel branch of the Bell Telephone Laboratories, would later become the mainstay of high-speed modulation of light in optical fiber communication [9].

During the late 1960s a couple of papers appeared that suggested the possibility of guiding and manipulating optical beams. The first such suggestion was made by Shubert and Harris in a 1968 paper [10] entitled "Optical surface waves on 
thin films and their application to integrated data processors." This was followed in 1969 by a paper by Miller of Bell Labs [11] "Integrated Optics: An introduction," which also suggested surface waveguiding as a basis for a new generation of devices for manipulating light. This is the first appearance, as far as I know, of the name "Integrated Optics," which would come to describe the field.

The Shubert and Harris paper as well as that of Miller provided the initial impetus to the field of Integrated Optics. A small symposium with that name was held in 1971 in Palo Alto, CA, organized by R. L. Byer of Stanford and O. Bryngdahl of Xerox, PARC. I took part in that symposium in which I described our Caltech work on guiding and modulation in GaAs. Unfortunately I no longer have the proceedings of the symposium, which can be considered as the first organized Integrated Optics meeting.

As many of the discussions in the new field turned to material aspects, it occurred to me that the III-V semiconductors which we were using at Caltech, primarily for guiding and modulation, offered a much broader platform to an Integrated Optics technology. This was due to the fact that one could now incorporate the principal actors of electronics: diodes and transistors with those of optics: waveguides, lasers, and modulators, and so on, monolithically on a single semiconductor crystal.

To distinguish this field from its older brother-Integrated Optics-I referred to it as "Active Integrated Optics." Later that year in a meeting in Esfahan, Iran, organized by A. Javan, I came back to the same topic. I will take here the liberty of reproducing a few paragraphs from the proceedings of the 1971 conference [12] which deal with the issue of integrated optoelectronic circuits.

“... just as silicon and germanium have come to play a key role in integrated electronics, it is possible already to develop approximate criteria which point toward certain materials as candidates for active integrated optics applications. Some of the more important requisite properties are

1) transparency and good optical quality for light in the visible and near-visible regions of the spectrum;

2) material should lend itself easily to interfacing with electronic circuits;

3) the material should be capable of light generation and detection;

4) the material should be capable of performing light switching and modulation functions. More specifically, it should possess large electrooptic and photoelastic figures of merit so that modulation and switching of light by either of these two techniques can be used;

5) the material should be suitable for thin-film dielectric waveguide fabrication. There are many materials that can satisfy reasonably well one or two of these requirements and it is conceivable that future integrated circuits will combine a number of them for specific applications. It is interesting to note, however, that at least one class of known materials already comes close to fulfilling all of these requirements. This is the semiconductor GaAs and its related alloys, such as $\mathrm{Ga}_{1-\mathrm{x}} \mathrm{Al}_{\mathrm{x}}$ As and $\mathrm{GaAs}_{1-\mathrm{x}} \mathrm{P}_{\mathrm{x}}$."

At the International Semiconductor Conference held in 1970 in Kyoto I gave a talk on the subject of Active Integrated Optics Circuits. After the talk I was approached in the conference hallway by a gentleman I had not met before. He introduced himself as Dr. Martin Stickley, a program director at Defense Advanced Research Projects Agency (DARPA), and offered to support a research project to realize my proposal of Integrated Optoelectronic Circuits [later to be known as optoelectronic integrated circuits (OEICs)]. Needless to say, I accepted this offer on the spot and have continued to work with DARPA to this date. Dr. Stickley retired from DARPA last year, and I told the above story during the retirement dinner.

At that time it became clear to us that to make further progress in this field, we would need to be able to grow our GaAs- $\mathrm{Ga}_{\mathrm{x}} \mathrm{Al}_{1-\mathrm{x}}$ As structures. This, at the time, seemed to be a scary excursion into the "black magic land" of crystal growth. With the help of I. Samid, a postdoctoral fellow from Israel, and Elsa Garmire we set up two liquid phase epitaxial reactors and by 1972 were growing our own crystals. In retrospect, this was a crucial decision for us since the increasing complexity of the devices which were required made it all but impossible, at the time, to get them from any other source but our own. It also pointed the way to the unavoidable need to develop at universities sophisticated semiconductor growth, fabrication, and test facilities in order to perform meaningful research and train students in a new and technologically demanding area.

A major impetus to our development of IOCs came when we started the fabrication of semiconductor lasers on semi-insulating substrates. This made it possible for us to adopt a planar technology where all contacts are made on top of the wafer so that different devices could be interconnected electrically. An example of an active device using this development was the integration of a Gunn diode oscillator and a laser diode [13], as shown in Fig. 3. The basic feature that makes this type of integration feasible is the layered epitaxial structure common to both the laser and Gunn diode. We note in the figure that the $\mathrm{GaAs}(\mathrm{N})$ channel doubles as the active region of the Gunn diode as well as serving as the interconnect "wire" that carries the microwave current output of the Gunn diode into the laser, thus modulating the latter at the microwave frequency. This simple combination dating to 1978 is, as far as I know, the first optoelectronic integrated circuit. In 1978, we also fabricated an OEIC which was closer to the world of communication which consisted of a buried heterostructure GaAs/GaAlAs laser fabricated monolithically with a field effect transistor [14]. The transistor's role was to supply and control the laser injection current. It is shown here in Fig. 4. Similar ideas must have occurred to a group of researchers at Bell Labs, and the above examples of the Caltech OEICs were followed with reports of P-I-N/FET integration in 1980 by Leheny et al. at Bell Labs [15], and by Fukuzawa et al. at Hitachi who in 1980 reported on the integration of an injection semiconductor laser with a Schottky-gate FET [16].

These initial efforts have clearly demonstrated the feasibility of monolithic integration of optical and electronic functions and the field has been growing for over 30 years. It is probably only during the last few years that OEICs are seriously being considered for mass markets.

If I am allowed to gaze into the crystal ball just once I would predict that OEICs will play an increasing role in optoelectronics technology starting in the near future. This will happen 


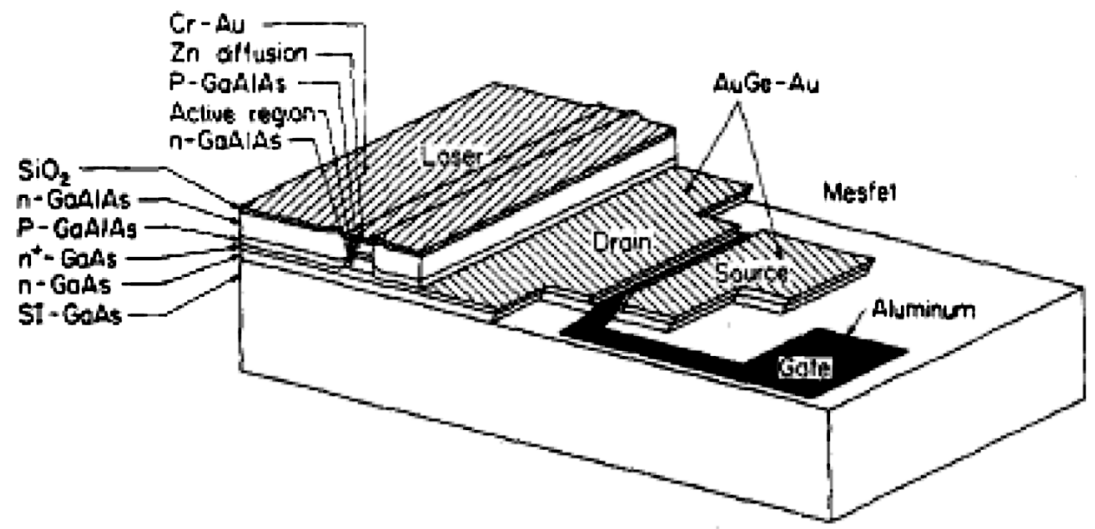

Fig. 4. Monolithic integration of a buried heterostructure GaAs/GaAlAs laser with a MESFET.

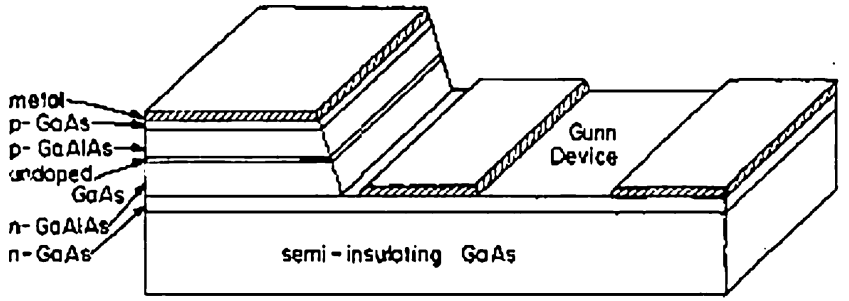

Fig. 3. Monolithic integration of a Gunn diode microwave oscillator with a laser diode.

mostly due to the relentless drive for extremely high data rate which will be delivered to the home. That prospect will become possible only when the cost of transceivers to the individual homes will drop down to the level of other mass communication components. This will demand a degree of integration and manufacturability which can only be satisfied by OEICs.

\section{ACKNOWLEDGMENT}

The author would like to acknowledge the many collaborators who as students and visitors to Caltech carried out the work described above. Major thanks are due to DARPA (M. Stickley), ONR (A. Shostak, L. Cooper), and AFOSR (H. Schlossberg) for the many years of support of our research in optoelectronics.

\section{REFERENCES}

[1] W. H. Louisell, A. E. Siegman, and A. Yariv, "Quantum fluctuations and noise in parametric processes," Phys. Rev., vol. 1, pp. 1646-, 1961.

[2] R. N. Hall, R. O. Carlson, T. J. Soltys, G. E. Fenner, and J. D. Kingsley, "Coherent light emission from GaAs junctions," Phys. Rev. Lett., vol. 9, pp. 366-, 1962.
[3] M. I. Nathan, W. P. Dumke, G. Burns, F. H. Dill, and G. Lasher, "Stimulated emission of radiation from GaAs p-n junctions," Appl. Phys. Lett., vol. 1, pp. 62-64, 1962.

[4] W. L. Bond, B. G. Cohen, R. C. C. Leite, and A. Yariv, "Observation of the dielectric-waveguide mode of light propagation in P-N junctions," Appl. Phys. Lett., vol. 2, pp. 57-59, 1963.

[5] R. C. C. Leite and A. Yariv, "On mode confinement in P-N junctions," Proc. IEEE, vol. 51, no. 7, Jul. 1963.

[6] A. Ashkin and M. Gershenzon, "Reflection and guiding of light at p-n junctions," J. Appl. Phys., vol. 34, pp. 2116-, 1963.

[7] Z. I. Alferov et al., "Investigation of the influence of the AlAs-GaAs heterostructure parameters on the laser threshold current and the realization of continuous emission at room temperature," Sov. Phys.-Semiconductors, vol. 4, pp. 1573-1575, 1971.

[8] D. Hall, A. Yariv, and E. Garmire, "Observation of propagation cutoff and its control in thin optical waveguides," Appl. Phys. Lett., vol. 17, pp. 127-, 1970.

[9] R. V. Schmidt and I. P. Kaminow, "Metal-diffused optical-waveguides in $\mathrm{LiNbO}_{3}, "$ Appl. Phys. Lett., vol. 25, pp. 458-460, 1974.

[10] R. Shubert and J. H. Harris, "Optical surface waves on thin films and their application to integrated data processors," IEEE Trans. Microw. Theory Tech., vol. MT16, pp. 1048-, 1968.

[11] S. E. Miller, "Integrated optics: An introduction," Bell Syst. Tech. J., vol. 48, pp. 2059-2069, 1969.

[12] A. Yariv, M. Feld, A. Javan, and N. Kurnit, Eds., "Active integrated optics," in Fundamental and Application Laser Physics, Proc. Esfahan 1971 Symp., 1973, pp. 597-921.

[13] C. P. Lee, S. Margalit, I. Ury, and A. Yariv, "Integration of an injectionlaser with a gunn oscillator on a semi-insulating GaAs substrate," Appl. Phys. Lett., vol. 32, pp. 806-807, 1978.

[14] I. Ury, S. Margalit, M. Yust, and A. Yariv, "Monolithic integration of an injection-laser and a metal-semiconductor field-effect transistor," Appl. Phys. Lett., vol. 34, pp. 430-431, 1979.

[15] R. F. Leheny, R. E. Nahory, M. A. Pollack, A. A. Ballman, E. D. Beebe, J. C. Dewinter, and R. J. Martin, "Integrated $\mathrm{In}_{0.53} \mathrm{Ga}_{0.47}$ As P-I-N FET photoreceiver," Electron. Lett., vol. 16, pp. 353-355, 1980.

[16] T. Fukuzawa, M. Nakamura, M. Hirao, T. Kuroda, and J. Umeda, "Monolithic integration of a GaAlAs injection-laser with a schottky-gate field-effect transistor," Appl. Phys. Lett., vol. 36, pp. 181-183, 1980

Amnon Yariv (S'56-M'59-F70-LF'95), photograph and biography not available at the time of publication. 